\title{
Detection of Ventricular Tachycardia Using Scanning Correlation Analysis
}

\author{
BRUCE M. STEINHAUS, RANDY T. WELLS, SAUL E. GREENHUT, * \\ STEVE M. MAAS, TIBOR A. NAPPHOLZ, JANICE M. JENKINS, * and \\ LORENZO A. DICARLO**
}

From Telectronics Pacing Systems, Englewood, Colorado; and *University of Michigan, Ann

Arbor, Michigan; and ** St. Joseph Mercy Hospital, Ann Arbor, Michigan

STEINHAUS, B.M., ET AL.: Detection of Ventricular Tachycardia Using Scanning Correlation Analysis. Cross correlation is an accurate method for distinguishing normal sinus rhythm (NSR) from ventricular arrhythmias. The computational demands of the method, however, have prohibited development of an implantable device using correlation. In this study, temporal data compression prior to correlation analysis was used to reduce the total number of computations. Unipolar and bipolar intracardiac electrograms of NSR and 23 episodes of ventricular tachycardia (VT) from 23 patients were obtained from a right ventricular apex electrode catheter during routine electrophysiology studies. The data were filtered (1$11 \mathrm{~Hz}$ ), digitized (250 samples/sec) and temporally compressed to $50 \mathrm{samples} / \mathrm{sec}$. Data compression removed four out of every five samples by only saving the sample with the maximum excursion from the last saved sample. The average squared correlation coefficient $\left(\mathrm{r}^{2}\right)$ was computed for the NSR and $V T$ episodes using each patient's NSR waveform as a template. In all 23 patients, the $r^{2}$ values showed large separation between NSR versus VT in both unipolar $[0.93 \pm 0.05$ vs $0.20 \pm 0.16, P<0.005)$ and bipolar $(0.91 \pm 0.07$ vs $0.17 \pm 0.11, \mathrm{P}<0.005)$ electrode configurations using template lengths of $80 \%$ the intrinsic interval (avg $\pm S D$ ). Narrow templates ( $40 \%$ intrinsic interval or less) often resulted in multiple $\mathrm{r}^{2}$ peaks during each heart cycle and degraded the $\mathrm{r}^{2}$ separation $(\mathrm{n}=10, \mathrm{P}<0.005)$. High pass filtering at $3 \mathrm{~Hz}$ also degraded the $\mathrm{r}^{2}$ separation $(\mathrm{n}=10, \mathrm{P}<0.05)$. Standard noncompressed correlations indicated that data compression had negligible effects on the results. Thus, a computationally efficient cross correlation method was found to be a reliable detector of VT. The results suggest that the T wave, as well as the QRS complex, is useful in VT recognition. (PACE, Vol. 13, December, Part II 1990)

ventricular tachycardia, correlation morphology analysis, antitachycardia device, data compression, ventricular repolarization

\section{Introduction}

Accurate detection of ventricular arrhythmias is of extreme importance for the safety of the patient with an implantable antitachycardia device. Typically, rate information is used to trigger the presence of the arrhythmia. Rate criteria alone, however, is not highly specific in identifying ventricular arrhythmias. Accurate arrhythmia detection is often improved when activation informa-

Address for reprints: Bruce M. Steinhaus, PhD, Research \& Development, Telectronics Pacing Systems, 7400 South Tucson Way, Englewood, CO 80112. tion from an atrial and/or multiple ventricular sites are used. ${ }^{1,2}$ The disadvantage of these methods is the requirement of one or more additional catheters. Single lead morphology analysis has typically concentrated on discrete features of the endocardial electrogram such as signal amplitude or slope. ${ }^{3.4}$ While these methods can often detect individual arrhythmia waveforms, the wide patient-to-patient variations in signal morphology could limit the utility of these methods.

Recently, correlation between a previously determined template waveform and a test waveform has been proposed as a morphology analysis tool. ${ }^{5}$ Correlation analysis is advantageous be- 
cause it provides a quantitative index of waveform similarity that is not dependent on any discrete waveform feature and the analysis is independent of differences in the signal baseline and amplitude. This study determined that data compression prior to correlation markedly reduced the computational demands of the method. Templates which included both the QRS and T wave were determined to improve the arrhythmia detection. These methods were studied in 23 patients and the results showed that the technique could provide a reliable and computationally efficient method to distinguish NSR from VT using endocardial electrogram morphology.

\section{Methods}

Data were collected from 23 patients undergoing electrophysiological studies. There were 18 men and 5 women of ages 24 to 86 (mean age of $65)$. Eleven patients had conduction abnormalities during NSR and the abnormalities were identified as either right bundle branch block, left bundle branch block, or intraventricular conduction defect. Fourteen patients had diagnosed coronary disease. The details of the electrogram data collection have been reported previously. ${ }^{6}$ Unipolar and bipolar right ventricular intracardiac electrograms were recorded from a temporary quadrapolar electrode catheter (USCI, Inc., Billerica, MA, USA) with $1-\mathrm{cm}$ interelectrode spacing. Unipolar signals from the distal electrode were referenced to a heparinized 0.035-inch guidewire (Cordis Corp., Miami, FL, USA) placed in the right femoral vein and positioned in the approximate location of current implanted antitachycardia devices. Intracardiac electrograms and body surface leads $V_{1}$, I, and III during NSR and episodes of induced monomorphic VT were amplified, filtered (1 to $500 \mathrm{~Hz}$, Pittsburgh Plate Glass, Pleasantville, NY, USA) and recorded using a FM tape recorder (Hewlett-Packard 3968A, San Diego, CA, USA). VT episodes lasted at least 30 seconds or required termination due to hemodynamic collapse.

\section{TEMPLATE LENGTH $=600 \mathrm{msec}$}
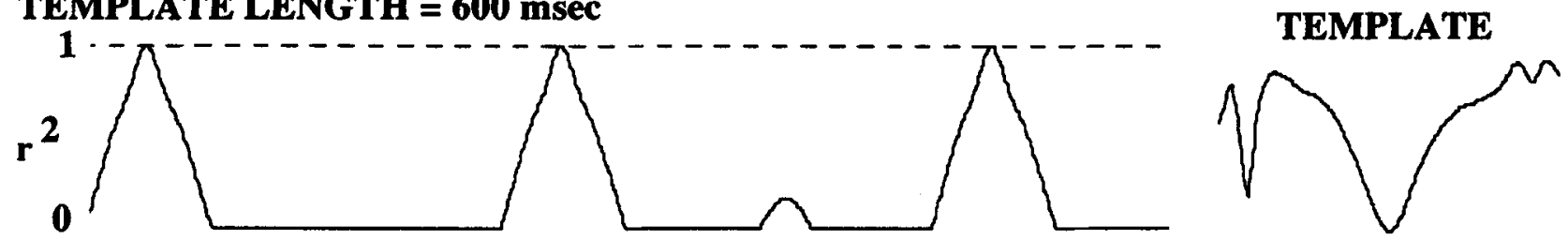

\section{TEMPLATE LENGTH $=100 \mathrm{msec}$}
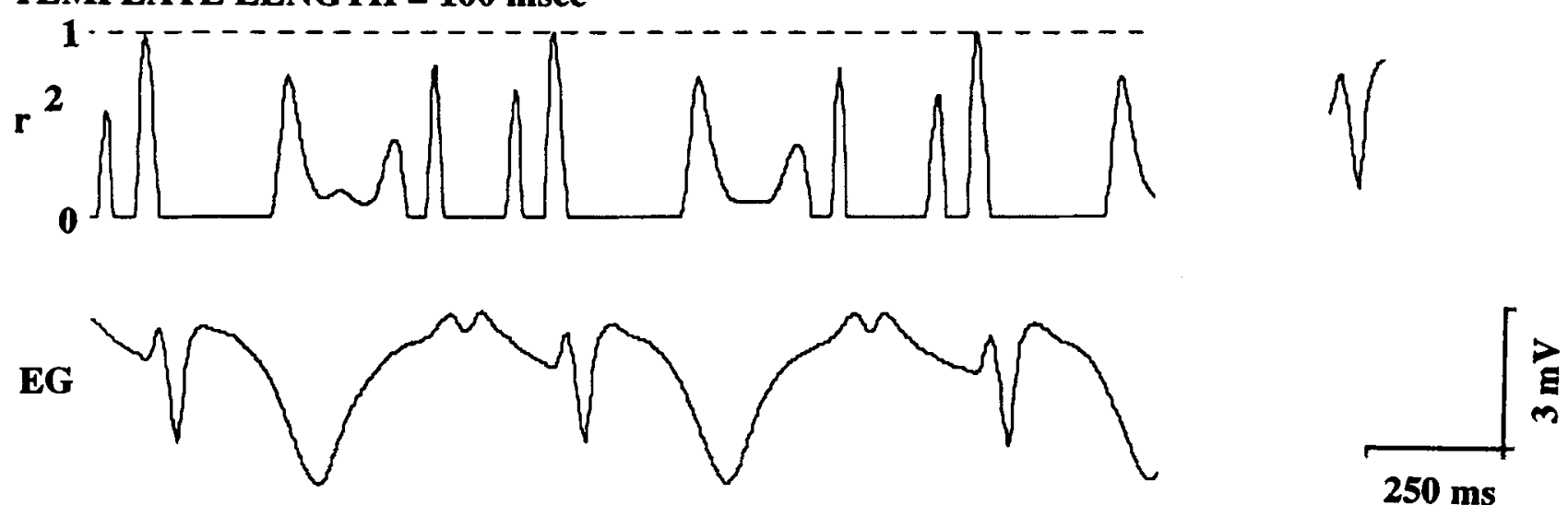

Figure 1. Scanning correlation results $\left(\mathrm{r}^{2}\right)$ using template lengths that include QRS and T wave (top panel) or QRS wave only (middle panel) from unipolar electrogram data during normal sinus rhythm (EG, bottom panel). The electrogram template morphologies used in the correlation analysis are shown to the right. Data sample rate is 1000 samples/sec. 
Data were copied onto a data instrumentation recorder (TEAC HR-30, Montebello, CA, USA) and digitized at 1000 samples/second using a $400 \mathrm{~Hz}$ antialiasing filter. Data were digitally low pass filtered at $125 \mathrm{~Hz}$ and decimated to 250 samples/sec to more closely model the sample rate available with current implantable technology. The data were then digitally low pass filtered at $11 \mathrm{~Hz}$ because initial results showed that this removed line frequency noise and had minor effects on the results. Additional results demonstrated that data compression to 50 samples/sec markedly reduced the required number of computations with minor effects on the results. Data compression from 250 samples/sec to $\mathbf{5 0}$ samples/sec used a custom nonlinear compression algorithm which saved the first sample and then analyzed a specified number of samples (five in this application) and determined the data sample with the maximum excursion from the last saved sample. That sample then became the next saved sample. Thus, four out of every five samples were removed from the input data resulting in an equivalent data rate of 50 samples/sec.

For each patient, the morphology of the electrogram recorded during VT was compared to a previously determined template of the electrogram recorded during NSR. Initial results showed that template lengths that included both the QRS and T-wave improved the arrhythmia detection (Fig. 1). Thus, templates were manually chosen to span approximately $80 \%$ of the NSR cycle length and ranged from $450 \mathrm{msec}$ to $700 \mathrm{msec}$. The NSR template was determined by first extracting a seed template of NSR. The template was then updated on each cycle with a correlation result $>0.9$. Each update was aligned to the time of the peak correlation and was given equal weight in the template average. This scheme allowed accommodation of the template to gradual changes in the morphology of the NSR waveform.

The correlation analysis used the following equation:

$$
r^{2}=\frac{\left(\sum_{i=0}^{N} T_{i} X_{i}\right)^{2}}{\sum_{i=0}^{N} T_{i}^{2} \sum_{i=0}^{N} X_{i}^{2}}
$$

where $T_{i}$ are the template samples, $X_{i}$ are the data samples, and $N$ is the number of template samples. Equation 1 differs from the standard correlation coefficient equation because initial results showed that the correction for the template and data mean values in the standard equation could be removed with only minor effect on the results. This finding was attributed to the previous removal of the mean value of the data via the $1-\mathrm{Hz}$ high pass filter. Computation of $\mathbf{r}^{2}$ instead of $r$ removed the square root computation in the standard correlation and also improved the arrhythmia detection. Values of $\mathrm{r}^{2}<0$ were set to zero. Scanning correlation analysis computed the results of Equation 1 at each data sample time and resulted in a time varying waveform of $r^{2}$ values.

The detection of VT was determined using the beat-to-beat peak $r^{2}$ values during the NSR and VT episodes. The beat-to-beat peak $\mathrm{r}^{2}$ values were automatically determined by iteratively comparing a threshold value to the time varying $\mathrm{r}^{2}$ values (Fig. $3)$. The $r^{2}$ threshold value was reduced until the number of beats via this technique was equal to the previously determined and verified number of beats in each data segment. The data segments of NSR averaged 103 beats (range: 34-332) and the segments of VT averaged 60 beats (range: $17-155$ ). The peak $\mathrm{r}^{2}$ average and standard deviation values were computed during NSR and VT and were used to determine a detection margin for each patient. The detection margin was determined by first computing the average of the peak $\mathrm{r}^{2}$ values during VT plus three times the standard deviation during VT. This value was then subtracted from the average of the peak $r^{2}$ values during NSR minus three times the standard deviation during NSR. Thus, a positive detection margin determined a confidence that at least $99.7 \%$ of all peak $\mathrm{r}^{2}$ values during VT cycles were less than at least $99.7 \%$ of all peak $\mathrm{r}^{2}$ values during NSR cycles. Signal analysis was performed on a micro-Vax II (Digital Equipment Corp., Marlboro, MA, USA) or IBM-PC compatible computer using custom software routines. Data are given as average value \pm standard deviation.

\section{Results}

The results of scanning correlation analysis comparing two different template lengths are 
shown in Figure 1. The lower panel shows the unipolar electrogram (EG) during NSR. The upper two panels show the time varying correlation coefficient values $\left(\mathrm{r}^{2}\right.$, Equation 1) using template lengths of $600 \mathrm{msec}$ and $100 \mathrm{msec}$. Correlation using a template length of $600 \mathrm{msec}$ included both QRS and $T$ wave morphology in the template and markedly removed the problem of multiple correlation peaks during the cycle. The multiple $r^{2}$ peaks shown in the middle panel of Figure 1 identified times throughout the cycle when the data was approximately proportional to the morphology of the template. As shown in the top panel of Figure 1, the longer template length reduced the probability of these occurrences. A disadvantage of the longer template lengths is an increase in the required number of computations.
Data compression prior to correlation markedly reduced the number of computations with minimal effect on the correlation results. Shown in Figure 2, top panel, are scanning correlation results and unipolar EG data using data sampled at 1000 samples/sec. The lower panel shows similar data following data compression to 50 samples/ sec. Data compression to $\mathbf{5 0}$ samples/sec resulted in a loss of low amplitude signal information and a temporal distortion which was most evident during the QRS complex where fast amplitude EG changes occurred. The compressed data, however, retained a majority of the morphology information in the original data. Thus, the $\mathrm{r}^{2}$ waveforms for the compressed and noncompressed data were similar. In this example, the 20 times data compression

\section{SAMPLES PER SECOND}

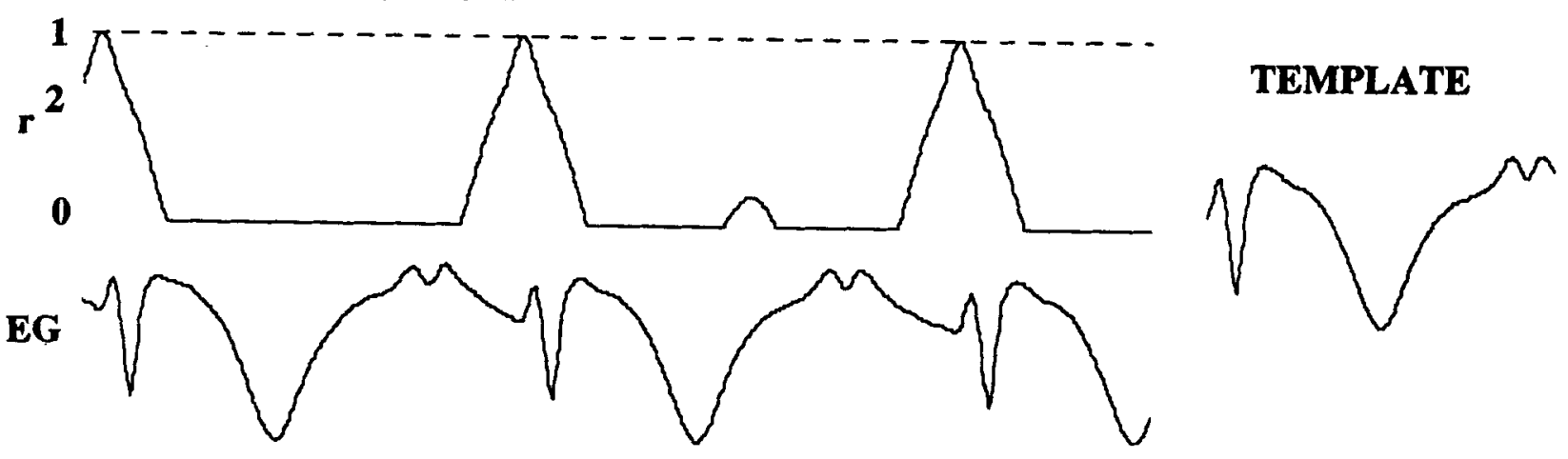

\section{O SAMPLES PER SECOND}

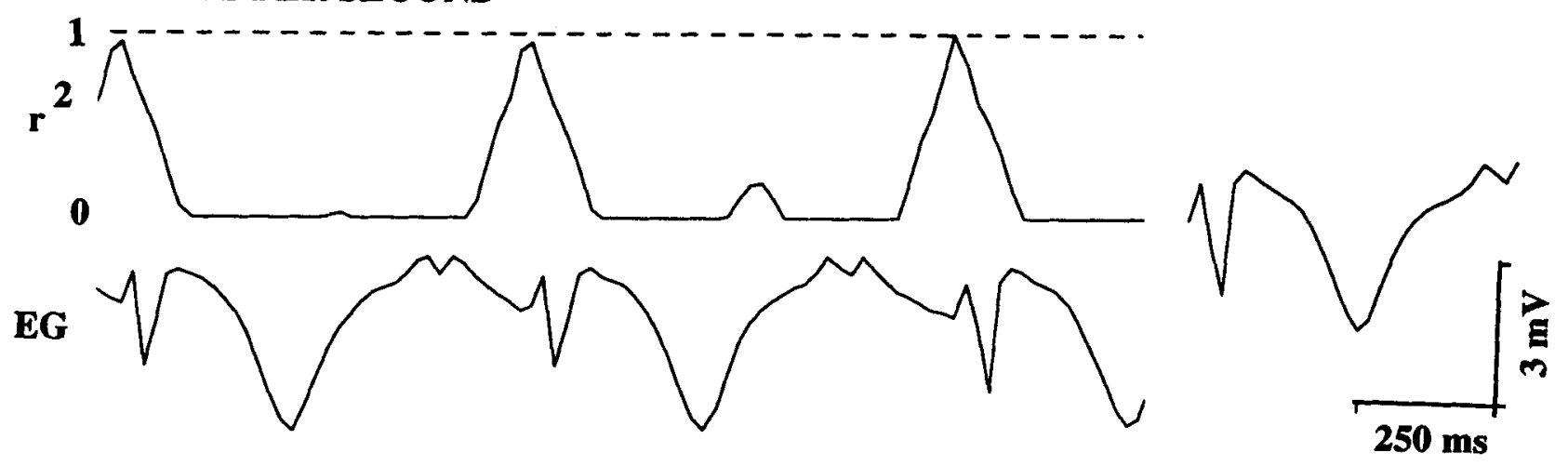

Figure 2. Scanning correlation results $\left(\mathrm{r}^{2}\right)$ and unipolar electrogram data (EG) comparing data sampled at 1000 samples/sec (top panel) and following data compression to 50 samples/sec (bottom panel). The electrogram template morphologies used in the correlation analysis are shown to the right. Data recorded during normal sinus rhythm. 
factor resulted in a 400 times reduction in computations.

Scanning correlation results during episodes of NSR and during induced monomorphic VT are shown in the top and bottom panels of Figure 3, respectively. The peak $\mathbf{r}^{2}$ value during each cycle was determined and plots of these data are shown to the right. For this patient, there was a large separation in the peak $\mathrm{r}^{2}$ values between NSR and VT. Peak $\mathrm{r}^{2}$ results for all 23 patients are shown in Figure 4 using the unipolar electrode configuration (panel A) and using the bipolar electrode configuration (panel B). The analysis method provided a wide separation in the average of the peak $\mathrm{r}^{2}$ values during NSR (filled triangles) and VT (filled circles) for each patient and also for the average of all patients (open triangles and circles). There was no significant difference between the results using unipolar or bipolar signals.

Additional studies investigated the change in the detection margin following removal of the $T$ wave from the template. In the first investigation, the data were digitally high pass filtered at $3 \mathrm{~Hz}$. In the 10 patients studied, this change degraded the $\mathrm{r}^{2}$ detection margin $(0.48 \pm 0.24$ vs $0.29 \pm$ $0.20, \mathrm{P}<0.05$, bipolar configuration; $0.40 \pm 0.28$ vs $0.22 \pm 0.20, \mathrm{P}<0.05$, unipolar configuration). In the second investigation, the template length was reduced to span only approximately $40 \%$ of the intrinsic interval (bandpass filter set at $1 \mathrm{~Hz}-$

\section{NSR}

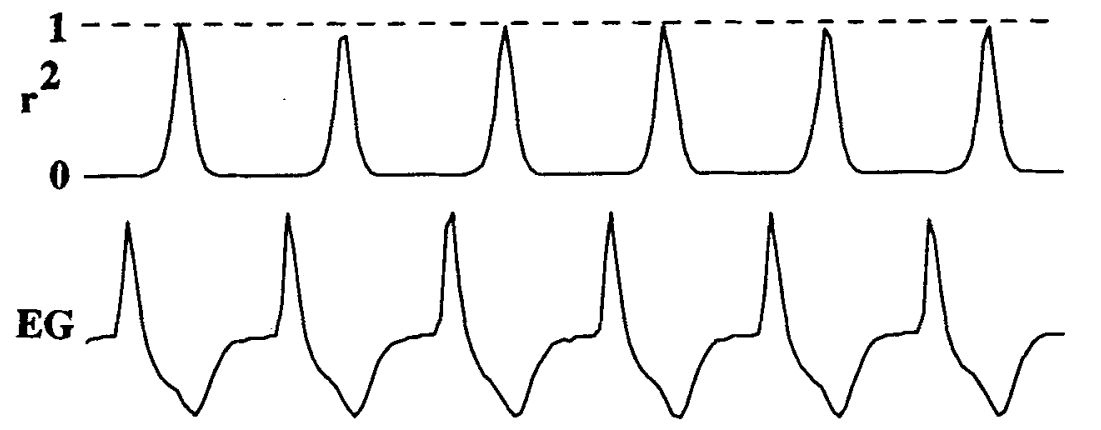

VT

$1 \ldots \ldots \ldots \ldots \ldots$ $\mathbf{r}^{2}$

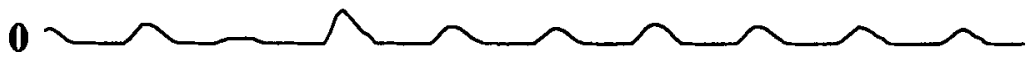

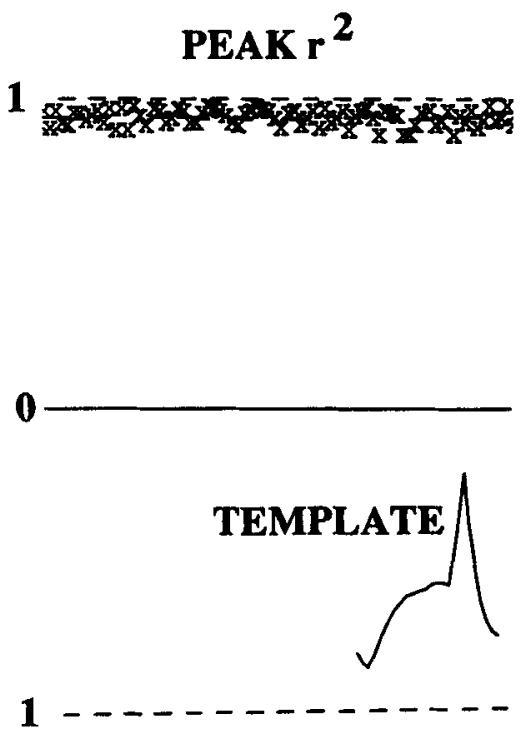

$\mathbf{1}$

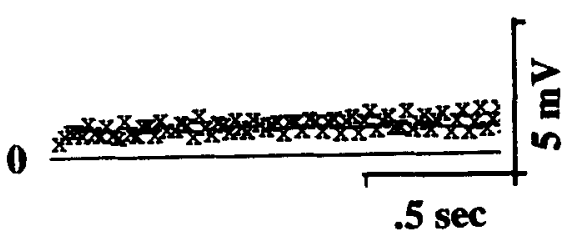

Figure 3. Scanning correlation results $\left(\mathrm{r}^{2}\right)$ during normal sinus rhythm (NSR, top panel) and during ventricular tachycardia (VT, bottom panel). Peak $\mathrm{r}^{2}$ data during NSR and VT are plotted vs time in the panels on the right. These results followed data compression to 50 samples/sec and used a 450 msec template obtained during NSR. Data are for patient number 3, bipolar configured signal. 

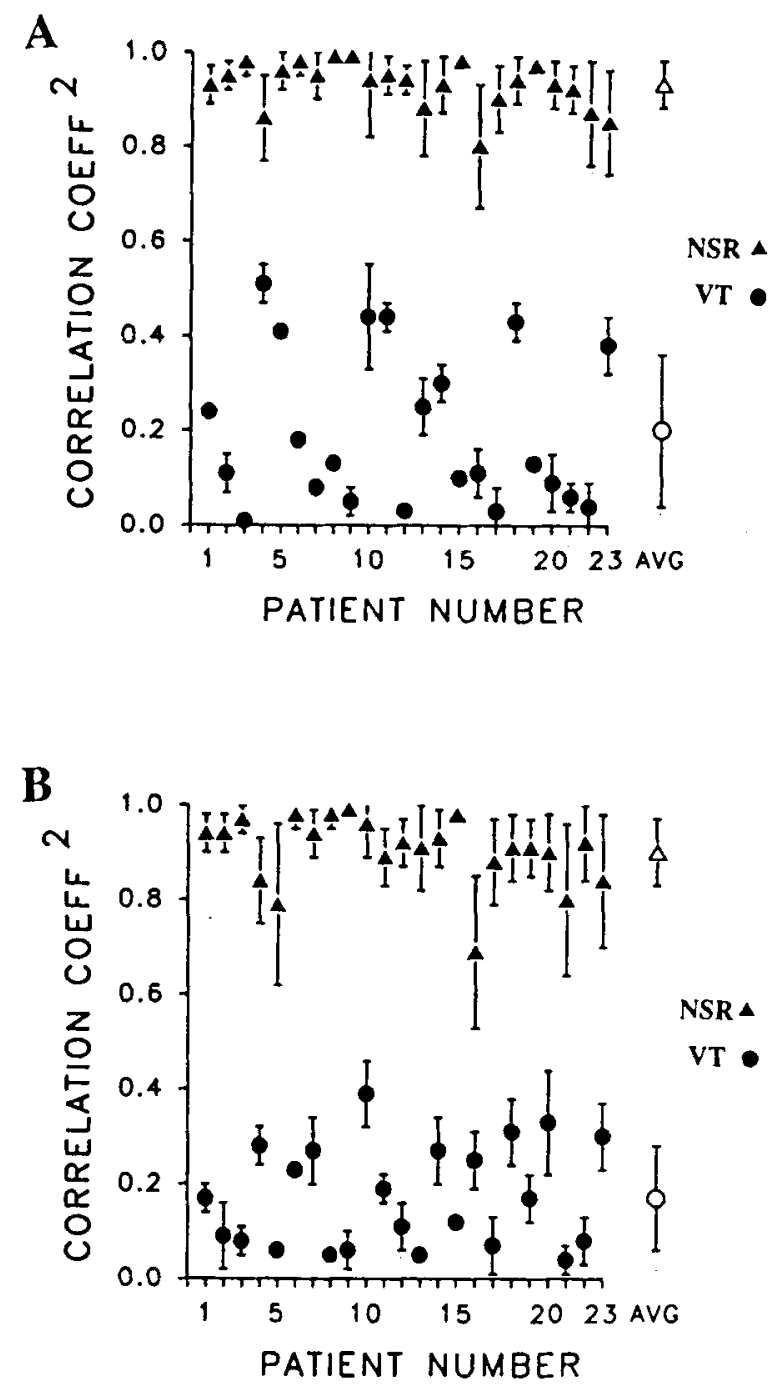

Figure 4. Correlation analysis results using unipolar configured signals (panel A) and bipolar configured signals (panel B) during NSR (filled triangles) and VT (filled circles) for each patient. The average of all patients was $0.93 \pm 0.05$ vs $0.20 \pm 0.16$ for unipolar NSR versus VT $(P<0.005)$ and $0.91 \pm 0.07$ vs $0.17 \pm 0.11$ for bipolar NSR versus VT $(P<0.005)$, respectively (open triangles and circles). The vertical bars signify the standard deviation for each data average.

$44 \mathrm{~Hz}$ ). In the same 10 patients, this change also degraded the $\mathrm{r}^{2}$ detection margin $[0.48 \pm 0.24$ vs $0.17 \pm 0.23, \mathrm{P}<0.005$, bipolar configuration; 0.40 \pm 0.28 vs $-0.12 \pm 0.29, \mathrm{P}<0.005$, unipolar configuration).

\section{Discussion}

This study investigated correlation analysis as a method to identify VT using right ventricular endocardial electrograms in 23 patients. Preliminary studies showed that minor beat-to-beat changes in waveform morphology could cause marked changes in correlation results when using externally triggered correlation techniques. This problem was attributed to beat-to-beat changes in the trigger alignment time. In contrast, the scanning correlation technique used in this study determined the peak of the time varying correlation values during each cycle. The scanning correlation method gave accurate correlation results during changes in the waveform morphology because it did not depend on an external hardware or software trigger. Data compression prior to correlation markedly reduced the numerical computations with minor effects on the detection margin. Another finding was that templates that included both ventricular activation and repolarization information markedly reduced the problem of multiple correlation peaks during the cycle. Electrogram analysis utilizing these findings provided a computationally efficient method to distinguish NSR from VT.

A previous study by Langberg et al. ${ }^{7}$ showed promising morphology analysis results using a normalized area of difference technique. The numerical requirements of this method are much less than for correlation, but the area of difference method has the disadvantage of being influenced by changes in either the baseline or amplitude of the signal. The amplitude of the endocardial electrogram has been shown to be influenced by multiple interventions, such as rate, exercise, cathecholimine infusion, ventricular volume, and Valsalva changes. ${ }^{8-11}$ Thus, the long-term reliability and detection ability of the area of difference method would be degraded in ambulatory patients.

This study compared unipolar and bipolar signals and found no overall preferred signal polarity. This result is consistent with other recent results. ${ }^{12}$ Of interest is that in any individual patient, there was often a preferred signal polarity to maximize the detection margin. This can be attributed to the difference in the lead field between unipolar and bipolar configurations, and the dif- 
ference in activation and recovery sequence during NSR and VT. Bipolar signals are insensitive to signals which are common to both electrodes used for the bipolar pair. Thus, bipolar electrograms are more likely to record local than distant cardiac events $^{13}$ and bipolar would be the preferred configuration if local activation and/or recovery were altered between NSR and VT. The unipolar electrogram, in contrast, contains distant information $^{14}$ and unipolar would be the preferred configuration if global activation and/or recovery were altered between NSR and VT. This study showed degradation in VT detection following high pass filtering of the data and also when using narrow templates. These interventions would tend to reduce the contribution of the $T$ wave. The degraded performance during these changes suggests that the $T$ wave, as well as the QRS complex, is useful information in VT recognition. Since the T wave is typically reduced in amplitude in bipolar electrograms, this finding would suggest that unipolar configuration would be preferred for VT detection.

The results of this study are promising, but

\section{References}

1. Mercando AD, Furman S, Kim SG, et al. Tachycardia differentiation using one atrial and two ventricular electrodes. (abstract) PACE 1987; 10:415.

2. Mercando AD, Furman S. Measurement of differences in timing and sequence between two ventricular electrodes as a means of tachycardia differentiation. PACE 1986; 9:1069-1078.

3. Davies DW, Wainwright RJ, Tooley MA, et al. Detection of pathological tachycardia by analysis of electrogram morphology. PACE 1986; 9:200-208.

4. Pannizzo F, Wanliss M, Furman S. Discriminator of ventricular tachycardia electrograms by syntactic methods. (abstract) J Am Coll Cardiol 1987; 9:98A.

5. Lin D, DiCarlo LA, Jenkins JM. Identification of ventricular tachycardia using intracavitary ventricular electrograms: Analysis of time and frequency domain patterns. PACE 1988; 11:15921606.

6. Throne RD, DiCarlo LA, Jenkins JM, et al. Paroxysmal bundle branch block of supraventricular origin: a possible source of misdiagnosis in detecting ventricular tachycardia using time domain analysis of intraventricular electrograms. PACE 1990; 13:453-468.

7. Langberg JJ, Gibb WJ, Auslander DM, et al. Identification of ventricular tachycardia with use of the morphology of the endocardial electrogram. Circulation 1988; 77:1363-1369. additional studies are necessary to demonstrate the practical implementation and limitations of the proposed method. One limitation of this study is that long template lengths necessarily contain rate information. As a consequence, the peak correlation results would be decreased at high rates and it is possible that correlation using long templates may be vulnerable to false detection of supraventricular tachycardia and sinus tachycardia as VT. Additional studies which allow for dynamic template length adjustment based on the detected heart rate are necessary. Future studies are necessary to develop and validate an automatic template acquisition and updating technique and an automatic VT detection criteria. Further studies are also necessary in determining the limitations of these methods in patients with intermittent conduction abnormalities. Clearly, morphology analysis should only be used in addition with other information available for rhythm identification and classification, such as rate, change in rate, rate stability, length of high rate, and hemodynamic status.

8. Lekven J, Chatterjee K, Tyberg JV, et al. Pronounced dependence of ventricular endocardial QRS potentials on ventricular volume. Br Heart J 1978 ; 40:891-901.

9. Rosenqvist $\mathrm{M}$, Lagergren $\mathrm{H}$, Strandberg $\mathrm{H}$, et al. Valsalva-induced variations in the intracardiac signal. PACE 1985; 8:856-861.

10. Bricker JT, Ward KA, Zinner A, et al. Decrease in canine endocardial and epicardial electrogram voltages with exercise: Implications for pacemaker sensing. PACE 1988; 11:460-464.

11. Callaghan F, Vollmann W, Livingston A, et al. The ventricular depolarization gradient: Effects of exercise, pacing rate, epinephrine, and intrinsic heart rate control on the right ventricular evoked response. PACE 1989; 12:1115-1130.

12. Greenhut SE, Throne RD, Jenkins JM, et al. A comparative analysis of unipolar versus bipolar intraventricular electrograms for detection of ventricular tachycardia. (abstract) PACE 1990; 13:496.

13. Steinhaus BM. Estimating cardiac transmembrane activation and recovery times from unipolar and bipolar extracellular electrograms: A simulation study. Circ Res 1989; 64:449-462.

14. Daniano RJ, Blanchard SM, Asano T, et al. Effects of distant potentials on unipolar electrograms in an animal model utilizing the right ventricular isolation procedure. J Am Coll Cardiol 1988; 11:1100-1109. 